\title{
First total synthesis of $(+)$-Neplanocin B
}

\author{
María J. Comin, ${ }^{a}$ Silvina C. Pellegrinet ${ }^{b}$, and Juan B. Rodriguez ${ }^{\text {a }}$ \\ ${ }^{a}$ Departamento de Química Orgánica, Facultad de Ciencias Exactas y Naturales, Universidad \\ de Buenos Aires, Pabellón 2, Ciudad Universitaria, C1428EHA, Buenos Aires, Argentina \\ ${ }^{b}$ Instituto de Química Orgánica y de Síntesis (CONICET), Facultad de Ciencias Bioquímicas y \\ Farmacéuticas, Universidad Nacional de Rosario, Suipacha 531, Rosario S2002LRK, Argentina \\ E-mail: jbr@qo.fcen.uba.ar
}

\section{Dedicated to Professor Rosa M. de Lederkremer on the occasion of her $\mathbf{7 0}^{\text {th }}$ birthday}

\begin{abstract}
$(+)$-Neplanocin B $((+)-5)$, the unnatural isomer of a minor component of the neplanocin family of antibiotics was enantioselectively synthesized starting from D-ribono-1,4-lactone. This synthetic strategy employed (+)-9-[(1R, 2R, 5S)-5-Benzyloxy-3-(benzyloxy)methyl-2-hydroxycyclopent-3-en-1-yl]-6-chloropurine (compound 7) as an advanced synthetic intermediate.
\end{abstract}

Keywords: Carbocyclic nucleosides; Neplanocin B; conformationally locked nucleosides; 6oxabicyclo[3.1.0]hexane; Ampullariella regularis

\section{Introduction}

The term carbanucleoside refers to a nucleoside analogue in which a methylene group has replaced the oxygen atom of the furanose ring. ${ }^{1-5}$ Surprisingly, these analogues are recognized by the same enzymes that recognize normal nucleosides exhibiting, in the same way, a broad range of pharmacological properties mainly as antiviral and antitumor agents. ${ }^{6,7}$ The neplanocin family is an important class of natural carbocyclic nucleosides isolated from Ampullariella regularis as fermentation products. ${ }^{7}$ Of the neplanocins, that include at least five components, such as neplanocin C ((-)-1), neplanocin A ((-)-2), neplanocin D ((-)-3), neplanocin F ((-)-4), and neplanocin B ((-)-5), only $\mathbf{1}$ and $\mathbf{2}$ have received much attention (Figure 1). 
<smiles>Nc1ncnc2c1ncn2[C@@H]1C[C@@]2(CO)C[C@H]1O[C@H]2O</smiles>

(-)-1, neplanocin C

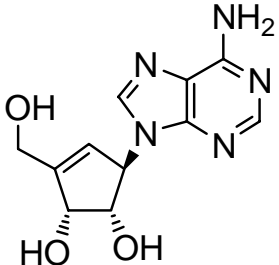

(-)-2, neplanocin A

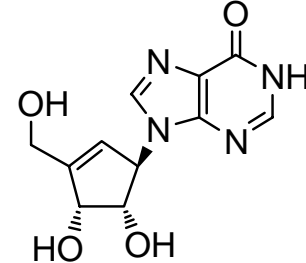

$(-)-3$, neplanocin D

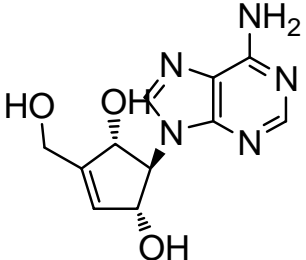

$(-)-4$, neplanocin F

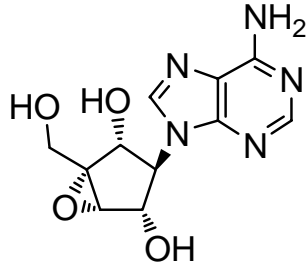

$(-)-5$, neplanocin B

Figure 1. Chemical structures of the neplanocin family of naturally occurring carbanucleosides.

The sugar moiety of nucleosides in solution exists in a dynamic equilibrium between the Northern-type geometry and the facing Southern-type geometry according to the concept of the pseudorotational cycle. ${ }^{8}$ However, only one of these conformers is found in the crystalline structure, and only the Northern or Southern conformer is exclusively responsible for molecular recognition as well, since only one form is present in the drug-enzyme complex. However, as the conformation in solution may be unlike that found in the solid state, any attempt to correlate sugar conformation with biological action would be flawed unless the crystal and the solution conformation are the same. A cyclopropane or an epoxy ring can confer such rigidity to the sugar ring, in which the equilibrium $\mathrm{N} \Leftrightarrow \mathrm{S}$ is not observed indicating that the solution conformation is practically the same to that found in the crystal. ${ }^{9}$ Neplanocin $C(\mathbf{1}),{ }^{10}$ is a good prototype of a conformationally locked nucleoside analogue, which is built on an oxabicyclic[3.1.0]-hexane system that allows this compound to exhibit the typical Northern-type $(\mathrm{N})$ conformation, specifically in the ${ }_{2} \mathrm{E}$ geometry. This conformation is very close to a pure ${ }^{3} \mathrm{~T}_{2}\left(\mathrm{P}=0^{\circ}\right)$ geometry as determined by the $P$ value of the pseudorotational cycle $\left(\mathrm{P}\right.$ value $=338.03^{\circ}$ and $\left.v_{\max }=21.89^{\circ}\right)$ from the solved $X$ ray structure. ${ }^{10 f}$ The chemical structure of neplanocin $\mathrm{C}$ was taken as lead drug for the design of a substantial number of conformationally restricted carbocyclic nucleosides of biological importance. ${ }^{11}$ The enantioselective synthesis of neplanocin $\mathrm{C}$ has been recently achieved employing D-ribonolactone as chiral starting material. ${ }^{12}$ The crucial point in the preparation of neplanocin $\mathrm{C}$ was the extraordinary stability of the epoxy group under methanolic ammonia at high temperature, which are the conditions required to convert a 6chloropurine derivative into an adenosine derivative. ${ }^{12}$ This fact has been used both to synthesize the corresponding $2^{2}$-deoxyneplanocin $\mathrm{C}$ analogues ${ }^{13}$ and to prepare simple and closely related carbanucleosides built in [3.1.0] oxabicyclo systems. ${ }^{14} \mathrm{~A}$ very interesting example constitutes a normal nucleoside built in a 2-oxabicyclic[3.1.0] hexane system. ${ }^{15}$ Taking nucleoside 6 as an example, this compound seems to be a closely related analogue of neplanocin B. Molecular modeling studies on $\mathbf{6}$ indicate that the optimized energy conformer presents a geometry close to a ${ }^{0} T_{1}$ conformation $\left(P=107.2^{\circ}\right)$ with a $v_{\max }=17.2^{\circ}$. The presence of a fused-three membered ring in this 2-oxabicyclo[3.1.0]hexane scaffold locks the sugar moiety in a C1'-exo type conformer according to the pseudorotational cycle. 


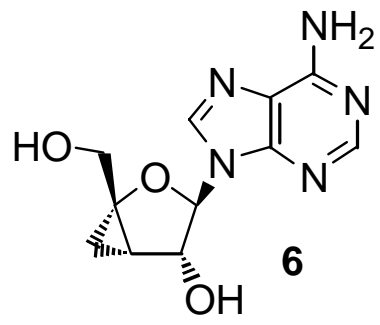

\section{Figure 2}

The lack of pharmacological activity data of neplanocin B encouraged us to develop an entry to access to carbanucleoside 5. Therefore, in this work we describe the first synthesis of the unnatural isomer of neplanocin B, which could be useful for the synthesis of analogues and closely related derivatives.

\section{Results and Discussion}

The synthesis of $(+)$-neplanocin B was successfully carried out employing compound (+)-9-[(1R, 2R, 5S)-5-benzyloxy-3-(benzyloxy)methyl-2-hydroxy-cyclopent-3-en-1-yl]-6-chloropurine (7), which in turn, was prepared from D-ribose in twelve steps. ${ }^{16}$ The adenosine precursor 7 was also employed as an advanced synthetic intermediate in the enantioselective synthesis of $(+)$ neplanocin F. ${ }^{16}$ Therefore, compound 7 was transformed into epoxy derivative $\mathbf{8}$ by treatment with $m$-chloroperbenzoic acid in $75 \%$ yield. The course of the epoxidation reaction was directed by the hydroxyl group and occurred with high stereoselectivity to afford a single diastereomer according to the Henbest rule. ${ }^{17}$ Formation of the diastereomeric $\beta$ epoxide was not observed. Treatment of $\mathbf{8}$ with methanolic ammonia gave the desired carbocyclic analogue of adenosine $\mathbf{9}$. In addition, no $\mathrm{N}$-oxide by-products were observed. Once again, the epoxy group was resistant to these strong basic conditions. Benzyl ethers cleavage with hydrogen in the presence of palladium on charcoal afforded the title compound, (+)-neplanocin B $((+)-5)$ in $70 \%$ yield for the first time. Compound numbering for all carbocyclic rings was kept for clarity in the NMR signal assignment, as illustrated for (+)-5 in Scheme 1. 


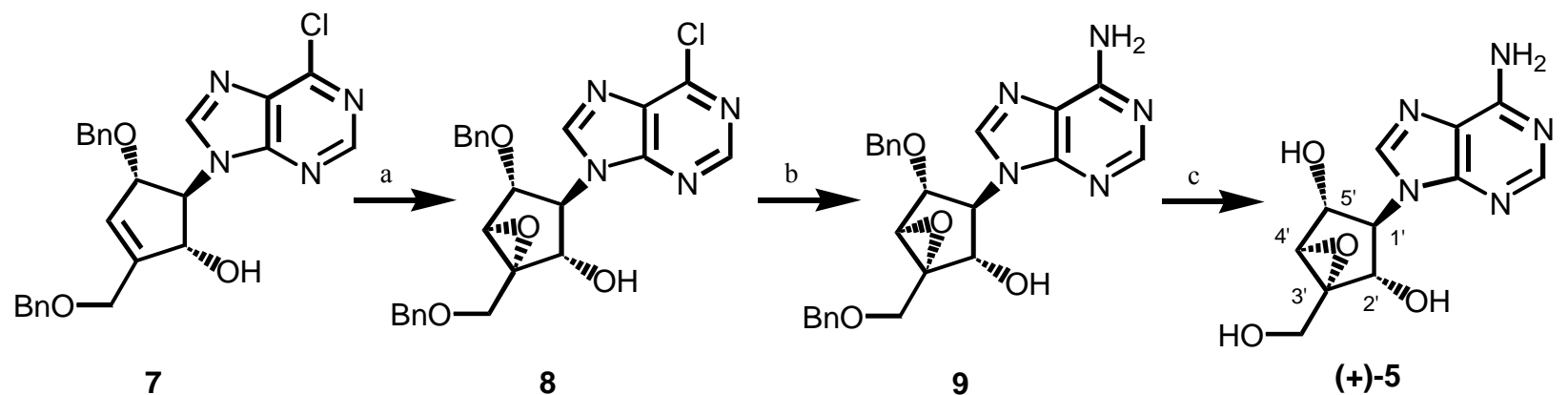

Scheme 1. Reagents and conditions: (a) m-CPBA, $\mathrm{CH}_{2} \mathrm{Cl}_{2}, 0{ }^{\circ} \mathrm{C} \rightarrow \mathrm{rt}, 48 \mathrm{~h}, 75 \%$; (b) methanol/ $\mathrm{NH}_{3}, 70{ }^{\circ} \mathrm{C}, 5 \mathrm{~h}, 60 \%$; (c) $\mathrm{H}_{2}, \mathrm{Pd} / \mathrm{C}$, methanol/HCOOH, $3 \mathrm{~atm}, 70 \%$.

In order to uncover the lowest energy conformer of neplanocin B ((+)-5), a conformational search was carried out. The initial conformations were generated by random variation of the dihedral angles using the conformational search protocol and the MM+ method as implemented in Hyperchem ${ }^{\mathrm{TM}}$ Release 7.52. A set of low-energy conformations were then minimized using $a b$-initio methods at the restricted Hartree-Fock (RHF) level of theory with the $3-21 \mathrm{G}$ basis set. $^{18}$ Finally, DFT optimizations using the B3LYP functional and the $6-31 \mathrm{G}^{*}$ basis set ${ }^{19}$ were performed on selected local minima. Frequency calculations were used to confirm the nature of the stationary points. Ab initio and DFT calculations were carried out using GAUSSIAN 98. ${ }^{20}$ The global minimum structure thus located has a south-type conformation possessing a ${ }_{1} \mathrm{E}$ geometry with $P=128.18^{\circ}$ and $v_{\max }=34.43^{\circ}$ (Figure 3). As was previously observed for nucleoside 6, the oxirane moiety of 5 appears to lock the conformation of the five-membered ring to a C1'-exo puckering mode. The greater stability of the global minimum relative to other ${ }_{1} \mathrm{E}$ conformers that have been found can be attributed to the presence of two intramolecular hydrogen bonds, as evidenced by the computed $\mathrm{O}-\mathrm{H}$ and $\mathrm{N}-\mathrm{H}$ distances depicted in Figure 3. Our calculations are supported by the relatively large experimental values obtained for $J_{12^{\prime}}$ of 5 (7.2$7.9 \mathrm{~Hz}$ ), which indicated that the studied compound should be effectively restricted to the Sform. $^{21}$

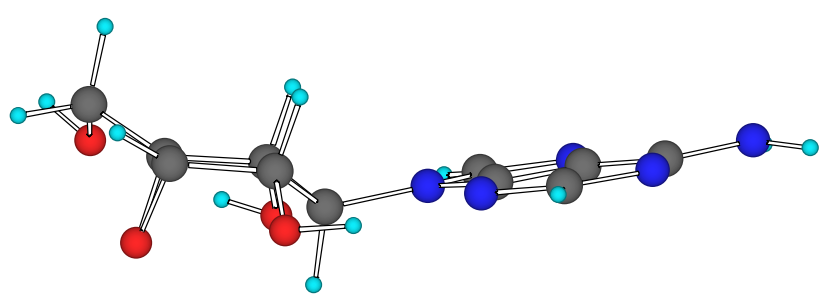

front view

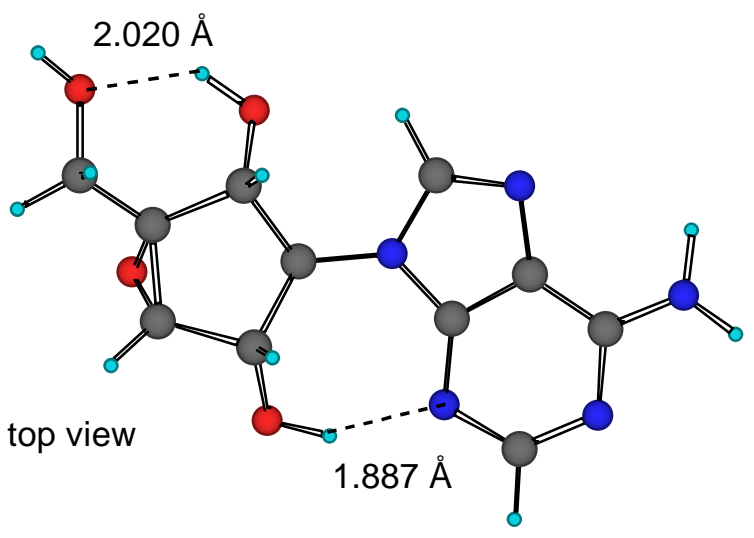

Figure 3. Lowest energy conformer of neplanocin B. 
In conclusion, we present the first total enantioselective synthesis of $(+)$-neplanocin B taking advantage of the availability of synthetic intermediate 7, which was employed for the enantioselective preparation of another member of neplanocin family, (+)-neplanocin F (4). Efforts aim at exploiting new synthetic methods to prepare neplanocin analogues are currently being pursued in our laboratory.

\section{Experimental Section}

General Procedures. The glassware used in air and/or moisture sensitive reactions was flamedried and carried out under a dry argon atmosphere. Unless otherwise noted, chemicals were commercially available and used without further purification.

Nuclear magnetic resonance spectra were recorded using a Bruker AM-500 MHz spectrometers. Chemical shifts are reported in parts per million $(\delta)$ relative to tetramethylsilane. The ${ }^{1} \mathrm{H}-\mathrm{NMR}$ spectra are referenced with respect to the residual $\mathrm{CHCl}_{3}$ proton of the solvent $\mathrm{CDCl}_{3}$ at 7.26 ppm. Coupling constants are reported in Hertz. ${ }^{13} \mathrm{C}$-NMR spectra were fully decoupled and are referenced to the middle peak of the solvent $\mathrm{CDCl}_{3}$ at $77.0 \mathrm{ppm}$. Splitting patterns are designated as s, singlet; d, doublet; $\mathrm{t}$, triplet; $\mathrm{q}$, quartet.

Melting points were determined using a Fisher-Johns apparatus and are uncorrected.

Positive ion fast atom bombardment mass spectra (FABMS) were obtained on a VG ZAB BEqQ spectrometer at an accelerating voltage of $8 \mathrm{kV}$ and a resolution of 500. Thioglycerol was used as the sample matrix, and ionization was effected by a beam of cesium atoms. Accurate mass analysis for the final products was conducted at a resolution of 3000 (10\% valley) in the molecular ion region using charge-exchanged xenon atoms and glycerol as a matrix. The glycerol peaks at m/z $277\left(\mathrm{Gly}_{3} \mathrm{H}^{+}\right), 369\left(\mathrm{Gly}_{4} \mathrm{H}^{+}\right)$and $461\left(\mathrm{Gly}_{5} \mathrm{H}^{+}\right)$were used as reference ions and the VG-7070-EHF mass spectrometer was voltage scanned under control of Maspec-II data system. Standard data system was used to assign and calculate masses for the reference and sample ions, respectively.

Column chromatography was performed with E. Merck silica gel (Kieselgel 60, 230-400 mesh). Analytical thin layer chromatography was performed employing $0.2 \mathrm{~mm}$ coated commercial silica gel plates (E. Merck, DC-Aluminum sheets, Kieselgel $60 \mathrm{~F}_{254}$ ) and was visualized by 254 $\mathrm{nm} \mathrm{UV}$ or by immersion into an ethanolic solution of $5 \% \mathrm{H}_{2} \mathrm{SO}_{4}$.

\section{(1S, 2S, 3R, 4R, 5R)-4-Benzyloxy-1-benzyloxymethyl-3-(6-chloropurin-9-yl)-6-oxabicyclo} [3.1.0]hexan-2-ol (8). To a solution of compound $7(120 \mathrm{mg}, 0.259 \mathrm{mmol})$ in methylene chloride $(5 \mathrm{~mL})$ was added dropwise a solution of $80 \% \mathrm{~m}$-chloroperbenzoic acid (179 $\mathrm{mg}, 0.51 \mathrm{mmol})$ in methylene chloride $(3 \mathrm{~mL})$ at $0{ }^{\circ} \mathrm{C}$. The reaction mixture was stirred at room temperature for 48 h. The solvent was evaporated and the residue was purified by column chromatography using a mixture of hexane-EtOAc (1:1) as the eluent to afford $91 \mathrm{mg}(75 \%$ yield) of pure epoxide 8 as a colorless oil: $R_{\mathrm{f}} 0.70$ (hexane-EtOAc, 4:1); ${ }^{1} \mathrm{H} \mathrm{NMR}\left(500.13 \mathrm{MHz}, \mathrm{CDCl}_{3}\right) \delta 3.75(\mathrm{~d}, J=1.1 \mathrm{~Hz}$, 
$\left.1 \mathrm{H}, \mathrm{H}-4{ }^{\prime}\right), 3.84$ (d, J=11.4 Hz, $\left.1 \mathrm{H}, \mathrm{CH}_{\mathrm{a}} \mathrm{HOBn}\right), 4.04$ (d, J=11.4 Hz, $\left.1 \mathrm{H}, \mathrm{CHH}_{\mathrm{b}} \mathrm{OBn}\right), 4.36$ (t, $\left.J=7.6 \mathrm{~Hz}, 1 \mathrm{H}, 1 \mathrm{H}, \mathrm{H}-1^{\prime}\right), 4.35$ (d, $\left.J=12.3 \mathrm{~Hz}, 1 \mathrm{H}, \mathrm{PhCH}_{\mathrm{a}} \mathrm{HO}\right), 4.62\left(\mathrm{~m}, 3 \mathrm{H}, \mathrm{PhCH}_{\mathrm{b}} \mathrm{O}\right.$, $\left.\mathrm{PhCH}_{2}\right), 4.70$ (dd, $\left.J=8.0,1.1 \mathrm{~Hz}, 1 \mathrm{H}, \mathrm{H}-5{ }^{\prime}\right), 5.03$ (d, $\left.J=7.5 \mathrm{~Hz}, 1 \mathrm{H}, \mathrm{H}-2^{\prime}\right), 6.92-7.05$ (m, 5 H, aromatic protons), 7.29-7.39 (m, $5 \mathrm{H}$, aromatic protons), 8.03 (s, $1 \mathrm{H}, \mathrm{H}-8), 8.45$ (s, 1 H, H-2); ${ }^{13} \mathrm{C}$ NMR (125.77 MHz, $\left.\mathrm{CDCl}_{3}\right) \delta 57.54\left(\mathrm{C}-1^{\prime}\right), 63.33\left(\mathrm{C}-3^{\prime}\right), 65.17\left(\mathrm{C}-4{ }^{\prime}\right), 67.32\left(\mathrm{CH}_{2} \mathrm{O}\right), 70.85$ $\left(\mathrm{C}-2^{\prime}\right), 71.80\left(\mathrm{CH}_{2} \mathrm{Ph}\right), 73.87\left(\mathrm{CH}_{2} \mathrm{Ph}\right), 75.38\left(\mathrm{C}-5{ }^{\prime}\right), 127,64(\mathrm{Ph}), 127.86(\mathrm{Ph}), 128.05(\mathrm{Ph})$, $128.13(\mathrm{Ph}), 128.53(\mathrm{Ph}), 136.67(\mathrm{Ph}), 137.34(\mathrm{Ph}), 146.04(\mathrm{C}-8), 152.17(\mathrm{C}-2)$.

(1S, 2S, 3R, 4R, 5R)-3-(6-Aminopurin-9-yl)-4-benzyloxy-1-benzyloxymethyl-6-oxabiciclo [3.1.0]hexan-2-ol (9). Compound 8 (100 $\mathrm{mg}, 0.209 \mathrm{mmol})$ was treated with methanolic ammonia $\left(5 \mathrm{~mL}\right.$, saturated at $-78^{\circ} \mathrm{C}$ ) and heated in sealed tube at $70{ }^{\circ} \mathrm{C}$ for $5 \mathrm{~h}$. The mixture was cooled to room temperature and the solvent was evaporated. The residue was purified by column chromatography (silica gel) using EtOAc as the eluent to afford $58 \mathrm{mg}$ (60\% yield) of pure 9 as a white solid: $R_{\mathrm{f}} 0.12$ (hexane-EtOAc, $\left.1: 9\right)$; mp $172{ }^{\circ} \mathrm{C}$; $[\alpha]^{24}{ }_{\mathrm{D}}+31.8$ (c 0.3, methanol); ${ }^{1} \mathrm{H}$ NMR (500.13 MHz, $\left.\mathrm{CDCl}_{3}\right) \delta 3.70$ (s, $1 \mathrm{H}, \mathrm{H}-4$ '), 3.80 (d, J=11.2 Hz, $1 \mathrm{H}, \mathrm{CH}_{\mathrm{a}} \mathrm{HOBn}$ ), 4.05 (d, $J=$ $11.4 \mathrm{~Hz}, 1 \mathrm{H}, \mathrm{CHH}_{\mathrm{b}} \mathrm{OBn}$ ), 4.29 (t, $\left.J=7.5 \mathrm{~Hz}, 1 \mathrm{H}, \mathrm{H}-1^{\prime}\right), 4.49$ (d, J=12.1 Hz, $1 \mathrm{H}, \mathrm{PhCH}_{\mathrm{a}} \mathrm{HO}$ ), 4.60 (d, $\left.J=7.7 \mathrm{~Hz}, 1 \mathrm{H}, \mathrm{H}-5^{\prime}\right), 4.62\left(\mathrm{~m}, 2 \mathrm{H}, \mathrm{PhCH}_{2}\right), 4.65$ (d, J = $\left.12.1 \mathrm{~Hz}, 1 \mathrm{H}, \mathrm{PhCHH}_{\mathrm{b}} \mathrm{O}\right)$, $4.93\left(\mathrm{~d}, J=7.3 \mathrm{~Hz}, 1 \mathrm{H}, \mathrm{H}-2^{\prime}\right), 5.83\left(\mathrm{~s}, 2 \mathrm{H}, \mathrm{NH}_{2}\right), 7.08-7.12$ (m, $2 \mathrm{H}$, aromatic protons), 7.147.18 (m, $3 \mathrm{H}$, aromatic protons), 7.28-7.36 (m, $5 \mathrm{H}$, aromatic protons), 7.64 (s, $1 \mathrm{H}, \mathrm{H}-8), 8.03$ (s, $1 \mathrm{H}, \mathrm{H}-2) ;{ }^{13} \mathrm{C}$ NMR (125.77 MHz, $\left.\mathrm{CDCl}_{3}\right) \delta 57.57$ (C-1'), 63.35 (C-3'), 64.19 (C-4'), 67.15 $\left(\mathrm{CH}_{2} \mathrm{O}\right), 70.94\left(\mathrm{C}-2^{\prime}\right), 71.52\left(\mathrm{CH}_{2} \mathrm{Ph}\right), 73.83\left(\mathrm{CH}_{2} \mathrm{Ph}\right), 76.65(\mathrm{C}-5 '), 119.97(\mathrm{C}-5), 127,71(\mathrm{Ph})$, $127.95(\mathrm{Ph}), 128.01(\mathrm{Ph}), 128.33(\mathrm{Ph}), 128.53(\mathrm{Ph}), 136.98(\mathrm{Ph}), 137.48(\mathrm{Ph}), 141.19(\mathrm{C}-8)$, 149.71 (C-4), 152.05 (C-2), 155.21 (C-6); HRMS (FAB) Calcd for $\mathrm{C}_{25} \mathrm{H}_{26} \mathrm{~N}_{5} \mathrm{O}_{4}\left(\mathrm{MH}^{+}\right) 460.1985$, found 460.1998 .

((+)-Neplanocin B, (1S, 2S, 3R, 4R, 5R)-(+)-3-(6-Aminopurin-9-yl)-1-hydroxymethyl-6oxabicyclo[3.1.0]hexane-2,4-diol ((+)-5). A solution of compound 9 (40 $\mathrm{mg}, 0.087 \mathrm{mmol})$ in methanol $(8 \mathrm{~mL})$ and formic acid $(0.4 \mathrm{~mL})$ in the presence of $5 \%$ palladium black $(5 \mathrm{mg}) \mathrm{was}$ treated with hydrogen at $3 \mathrm{~atm}$. The reaction was stirred at room temperature for $4 \mathrm{~h}$. The mixture was filtered off and the solvent was evaporated. The residue was purified by column chromatography (silica gel) employing a mixture of $\mathrm{CH}_{2} \mathrm{Cl}_{2}-\mathrm{MeOH}$ (4:1) as eluent to afford 17 $\mathrm{mg}\left(70 \%\right.$ yield) of pure (+)-5 as a white solid: $\mathrm{mp}>270{ }^{\circ} \mathrm{C}$ (lit. ${ }^{10 \mathrm{e}} \mathrm{mp} 269-272{ }^{\circ} \mathrm{C}$, decomp.); $[\alpha]^{24}+2.1$ (c 0.3, DMSO), it. $^{10 \mathrm{e}}[\alpha]_{\mathrm{D}}^{24}-3.5$ (c 1.0, DMSO) for the natural isomer; UV (MeOH) $\lambda_{\max } 260 \mathrm{~nm} ;{ }^{1} \mathrm{H}$ NMR (500.13 MHz, DMSO-d $\left.)_{6}\right) 3.51$ (s, $1 \mathrm{H}, \mathrm{H}-4$ '), 3.52 (dd, J=11.8, $5.0 \mathrm{~Hz}$, $1 \mathrm{H}, \mathrm{CH}_{\mathrm{a}} \mathrm{HOH}$ ), 3.88 (dd, $\left.J=12.4,6.5 \mathrm{~Hz}, 1 \mathrm{H}, \mathrm{CH}_{\mathrm{b}} \mathrm{HOH}\right), 4.06$ (t, $\left.J=7.9 \mathrm{~Hz}, 1 \mathrm{H}, \mathrm{H}-1^{\prime}\right), 4.63$ (t, $\left.J=7.2 \mathrm{~Hz}, 1 \mathrm{H}, \mathrm{H}-5^{\prime}\right), 4.70$ (dist t, $\left.J=7.2 \mathrm{~Hz}, 1 \mathrm{H}, \mathrm{H}-2^{\prime}\right), 4.85$ (t, $\left.J=5.8 \mathrm{~Hz}, 1 \mathrm{H}, \mathrm{OH}\right), 5.43$ $(\mathrm{d}, J=6.4 \mathrm{~Hz}, 1 \mathrm{H}, \mathrm{OH}), 5.47(\mathrm{~d}, J=5.7 \mathrm{~Hz}, 1 \mathrm{H}, \mathrm{OH}), 7.15$ (s, $2 \mathrm{H}, \mathrm{NH}$ ), 8.07 (s, 1 H, H-8), 8.10 (s, $1 \mathrm{H}, \mathrm{H}-2) ;{ }^{13} \mathrm{C}$ NMR $\left(125.77 \mathrm{MHz}, \mathrm{DMSO}-d_{6}\right) \delta 57.59\left(\mathrm{C}-1^{\prime}\right), 59.18\left(\mathrm{CH}_{2} \mathrm{OH}\right), 64.61(\mathrm{C}-$ 3'), 64.72 (C-4'), 69.66 (C-2'), 69.69 (C-5'), 119.94 (C-5), 141.76 (C-8), 149.74 (C-4), 151.91 (C2), 156.02 (C-6); HRMS (FAB) Calcd for $\mathrm{C}_{11} \mathrm{H}_{14} \mathrm{~N}_{5} \mathrm{O}_{4}\left(\mathrm{MH}^{+}\right)$280.1046, found 280.1042 . 


\section{Acknowledgements}

This work was supported by grants from the Fundación Antorchas, and the Universidad de Buenos Aires (X-252). We also thank Dr. James A. Kelley, Laboratory of Medicinal Chemistry, NCI, National Institutes of Health for accurate mass determination for the final product. SCP thanks CONICET, Universidad Nacional de Rosario, Fundación Antorchas and ANPCyT.

\section{References and Footnotes}

1. Crimmins, M. T. Tetrahedron 1998, 54, 9229.

2. Rodriguez, J. B.; Comin, M. J. Mini Rev. Med. Chem. 2003, 3, 95.

3. Borthwick, A. D.; Biggadike, K. Tetrahedron 1992, 48, 571.

4. Agrofolio, L.; Suhas, E.; Farese, A.; Condom, R.; Challand, S. R.; Earl, R. A.; Guedj, R. Tetrahedron 1994, 50, 10611.

5. Zhu, X. F. Nucleosides Nucleotides Nucleic Acids 2000, 19, 651.

6. Marquez, V. E.; Lim, M. -I. Med. Res. Rev. 1986, 6, 1.

7. Marquez, V. E. In Advances in Antiviral Drug Design; De Clercq, E. Ed.; JAI Press Inc.: Greenwich, CT, 1996; Vol 2, pp 89-146.

8. Saenger, W. Principles of Nucleic Acid Structure; Springer-Verlag: New York, 1984; pp 51-104.

9. Koole, L. H.; Neidle, S.; Crawford, M. D.; Krayevski, A. A.; Gurskaya, G. V.; Sandström, A.; Wu, J.-C.; Tong, W.; Chattopadhyaya, J. J. Org. Chem. 1991, 56, 6884.

10. (a) Yaginuma, S.; Muto, N.; Tsujino, M.; Sudate, Y.; Hayashi, M.; Otani, M. J. Antibiot. 1981, 34, 359. (b) Isono, K. J. Antibiot. 1988, 41, 1711. (c) De Clercq, E. Antimicrob. Agents Chemother. 1985, 28, 84. (d) De Clercq, E.; Bernaerts, R.; Bregstrom, D. E.; Robins, M. J.; Montgomery, J. A.; Holy, A. Antimicrob. Agents Chemother. 1986, 29, 482. (e) Hayashi, M.; Yaginuma, S.; Muto, N.; Tsujino, M. Nucleic Acids Res. Symp. Ser. 1980, 8, s65. (f) Kinoshita, K.; Yaginuma, S.; Hayashi, M.; Nakatsu, K. Nucleosides Nucleotides 1985, 4, 661 .

11. (a) Rodríguez, J. B.; Márquez, V. E.; Nicklaus, M. C.; Barchi, J. J., Jr. Tetrahedron Lett. 1993, 34, 6233. (b) Rodriguez, J. B.; Marquez, V. E.; Nicklaus, M. C.; Mitsuya, H.; Barchi, J. J., Jr. J. Med. Chem. 1994, 37, 3389. (c) Marquez, V. E.; Ezzitouni, A.; Russ, P.; Siddiqui, M. A.; Ford, H. Jr.; Feldman, R. J.; Mitsuya, H.; George, C.; Barchi, J. J., Jr. J. Am. Chem. Soc. 1998, 120, 2780. (d) Siddiqui, M. A.; Ford, H., Jr.; George, C.; Marquez, V. E. Nucleosides Nucleotides 1996, 15, 235. (e) Marquez, V. E.; Siddiqui, M. A.; Ezzitouni, A.; Russ, P.; Wang, J.; Wagner, R. W.; Mateucci, M. D. J. Med. Chem. 1996, 39, 3739. (f) Altmann, K. -H.; Kesselring, R.; Francotte, E.; Rihs, G. Tetrahedron Lett. 1994, 35, 2331. (g) Altmann, K. -H.; Imwinkelried, R.; Kesselring, R.; Rihs, G. Tetrahedron Lett. 1994, 35, 7265. (h) Marquez, V. E.; Russ, P.; Alonso, R.; Siddiqui, M. A.; Shin, K. -J.; George, C.; 
Nicklaus, M. C.; Dai, F., Ford, Jr., H. Nucleosides Nucleotides 1999, 18, 521. (i) Jeong, L. S.; Marquez, V. E.; Yuan, C. -S.; Borchardt, R. T. Heterocycles 1995, 41, 2651. (j) Ezzitouni, A.; Marquez, V. E. J. Chem. Soc., Perkin Trans 1 1997, 1073. (k) Jeong, L. S.; Buenger, G.; McCormack, J. J.; Cooney D. A.; Hao, Z.; Marquez, V. E. J. Med. Chem. 1998, 41, 2572. (1) Ezzitouni, A.; Russ, P.; Marquez, V. E. J. Org. Chem. 1997, 62, 4870-4873. (m) Moon, H. R.; Ford, H. Jr.; Marquez, V. E. Org. Lett. 2000, 24, 3793. (n) Shin, K. J.; Moon, H. R.; George, C.; Marquez, V. E. J. Org. Chem. 2000, 65, 2172. (o) Moon, H. R.; Kim, H. O.; Chun, M. W.; Jeong, L. S.; Marquez, V. E. J. Org. Chem. 1999, 64, 4733. (p) Lee, J. A.; Moon, H. R.; Kim, H. O.; Kim, K. R. Lee, K. M.; Kim, B. T.; Hwang, K. J.; Chun, M. W.; Jacobson, K. A.; Jeong, L. S. J. Org. Chem. 2005, 70, 5006. (q) Tchilibon, S.; Joshi, B. V.; Kim, S. -K.; Duong, H. T.; Gao, Z. -G.; Jacobson, K. A. J. Med. Chem. 2005, 48, 1745. (r) Joshi, B. V.; Moon, H. R.; Fettinger, J. C.; Marquez, V. E.; Jacobson, K. A. J. Org. Chem. 2005, 70, 439. (s) Gao, Z. -G.; Jeong, L. S.; Moon, H. R.; Kim, H. O.; Choi, W. J.; Shin, D. H.; Elhalem, E.; Comin, M. J.; Melman, N.; Mamedova, L.; Gross, A. S.; Rodriguez; J. B.; Jacobson, K. A. Biochem. Pharmacol. 2004, 67, 893. (t) Kim, H. S.; Ravi, R. G.; Marquez, V. E.; Maddileti, S.; Wihlborg, A.-K.; Erlinge, D.; Malsmjo, M.; Boyer, J. L.; Harden, T. K.; Jacobson, K. A. J. Med. Chem. 2002, 45, 208.

12. Comin, M. J.; Rodriguez, J. B. Tetrahedron 2000, 56, 4639.

13. Comin, M. J.; Rodriguez, J. B.; Russ, P.; Marquez, V. E. Tetrahedron 2003, 59, 295.

14. Comin, M. J.; Pujol, C. A.; Damonte, E. B.; Rodriguez, J. B.; Nucleosides Nucleotides 1999, $18,2219$.

15. Gagneron, J.; Gosselin, G.; Mathé, C. J. Org. Chem. 2005, 70, 6891.

16. Comin, M. J.; Leitofuter, J.; Rodriguez, J. B. Tetrahedron 2002, 58, 3129.

17. Henbest, H. B.; Wilson, R. A. L. J. Chem. Soc. 1957, 1958.

18. Hehre, W. J.; Radom, L.; Schleyer, P. v. R.; Pople, J. A. Ab Initio Molecular Orbital Theory; Wiley: New York, 1986.

19. (a) Becke, A. D. J. Chem. Phys. 1993, 98, 5648. (b) Lee, C.; Yang, W.; Parr, R. Phys. Rev. B 1988, 37, 785 .

20. Gaussian 98, Revision A.7, Frisch, M. J.; Trucks, G. W.; Schlegel, H. B.; Scuseria, G. E.; Robb, M. A.; Cheeseman, J. R.; Zakrzewski, V. G.; Montgomery, J. A. Jr.; Stratmann, R. E.; Burant, J. C.; Dapprich, S.; Millam, J. M.; Daniels, A. D.; Kudin, K. N.; Strain, M. C.; Farkas, O.; Tomasi, J.; Barone, V.; Cossi, M.; Cammi, R.; Mennucci, B.; Pomelli, C.; Adamo, C.; Clifford, S.; Ochterski, J.; Petersson, G. A.; Ayala, P. Y.; Cui, Q.; Morokuma, K.; Malick, D. K.; Rabuck, A. D.; Raghavachari, K.; Foresman, J. B.; Cioslowski, J.; Ortiz, J. V.; Stefanov, B. B.; Liu, G.; Liashenko, A.; Piskorz, P.; Komaromi, I.; Gomperts, R.; Martin, R. L.; Fox, D. J.; Keith, T.; Al-Laham, M. A.; Peng, C. Y.; Nanayakkara, A.; Gonzalez, C.; Challacombe, M.; Gill, P. M. W.; Johnson, B.; Chen, W.; Wong, M. W.; Andres, J. L.; Gonzalez, C.; Head-Gordon, M.; Replogle, E. S.; Pople, J. A. Gaussian, Inc.: Pittsburgh PA, 1998. 
21. (a) Obika, S.; Andoh, J. -I.; Onoda, M.; Nakagawa, O.; Hiroto, A.; Sugimoto, T.; Imanishi, T. Tetrahedron Lett. 2003, 44, 5267. (b) Obika, S.; Morio, K. -I.; Nanbu, D.; Hari, Y.; Itoh, H.; Imanishi, T. Tetrahedron 2002, 58, 3039 and references cited therein. 